\title{
Entwicklungsbiologie
}

\section{Wie Tiere sich selbst konstruieren}

DANIEL ČAPEK, MURAT ÜNALAN, PATRICK MÜLLER

FRIEDRICH-MIESCHER-LABORATORIUM DER MAX-PLANCK-GESELLSCHAFT, TÜBINGEN FACHBEREICH BIOLOGIE, UNIVERSITÄT KONSTANZ

The central question in developmental biology is how an embryo self-organizes from a ball of cells into a structured animal. We use experimental and theoretical approaches to study how the activity of a small number of signaling molecules is spatiotemporally controlled to allow for embryonic self-construction. Here, we propose how machine learning could be harnessed to gain a new understanding of the mechanisms by which interactions between signaling pathways control robust embryogenesis and morphology changes in evolution.

DOI: $10.1007 / \mathrm{s} 12268-021-1622-4$

(C) Die Autoren 2021

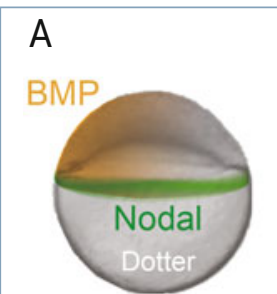

$\mathrm{C}$

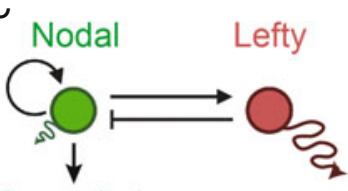

Mesendoderm

$\mathrm{E}$

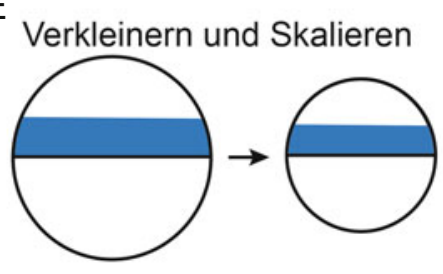

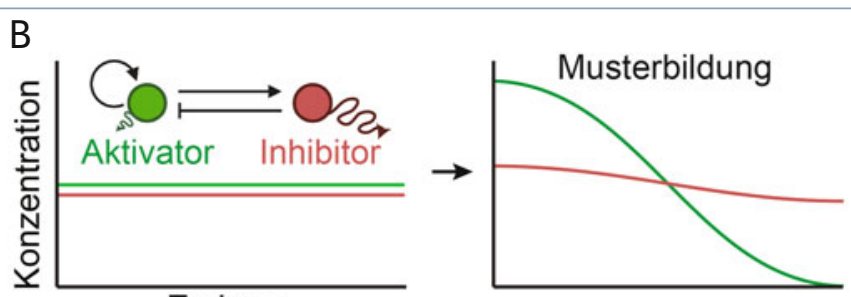

Embryo

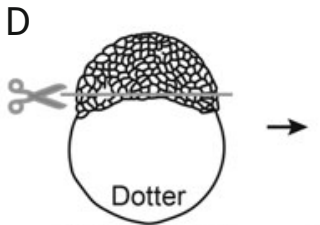

Explantieren

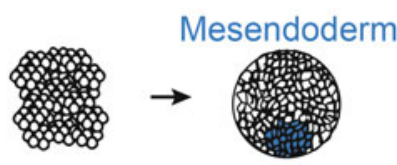

Dissoziieren Reaggregieren

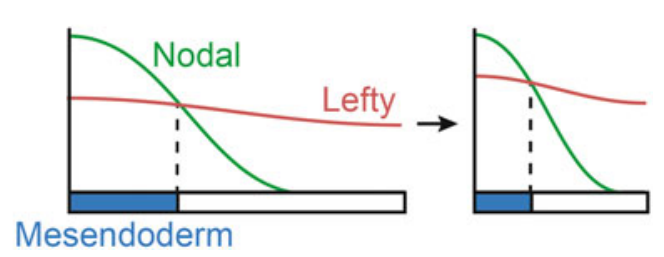

$\Delta$ Abb. 1: Embryonale Musterbildung durch Morphogene. A, Beim Zebrafisch befinden sich die Zellen des eigentlichen Embryos auf der oberen Ei-Hälfte, wohingegen die untere Ei-Hälfte als Dottersack der Versorgung dient. Ähnlich einem Koordinatensystem unterteilen Nodal und BMP das Zellfeld durch orthogonale Konzentrationsgefälle in unterschiedliche Bereiche. B, Aus einem anfangs homogenen Gemisch entwickelt sich durch die Regeln der spontanen Selbstorganisation ein Muster des Aktivator-Inhibitor-Systems, und Zellen können ihre Lage eindeutig anhand der lokalen Konzentration der Moleküle erkennen. C, Nodal und Lefty bilden genau solch ein Aktivator-Inhibitor-System, das während der Embryonalentwicklung Mesendoderm induziert. D, Durchmischt man durch Dissoziation und Reaggregation die Lage aller Zellen eines Zebrafischembryos, lässt sich ein Symmetriebruch mit polarisierter Mesendodermbildung (blau) erneut beobachten. E, Größenregulation durch den langreichweitigen Inhibitor Lefty: In einem künstlich verkleinerten Embryo steigt die Konzentration des Inhibitors, wodurch sich die Nodal-Signalintensität und folglich das Verhältnis der Keimblätter an die neue Größe anpasst.
Für Wissenschaftler ist die Embryonalentwicklung eine Goldgrube. Zellen zeigen im Embryo von Wachstum und Differenzierung bis zur kollektiven Migration ganzer Zellpopulationen fast alle ihre Fähigkeiten. Die regulatorischen Aspekte sind mindestens ebenso vielfältig, Mechanik genauso wichtig wie genetische und epigenetische Kontrolle. Außerdem lassen sich all diese Vorgänge gut beobachten, da Embryonen für gewöhnlich klein und häufig transparent sind. Und da viele Prozesse stereotyp ablaufen, lassen sich die Beobachtungen beinahe beliebig wiederholen - als würde man einen Film nochmal von vorne starten. Kein Wunder also, dass sich Wissenschaftler aus unterschiedlichsten Disziplinen - von der Biochemie bis zur Physik - die Entwicklung der Tiere als ihr Modellsystem ausgesucht haben.

\section{Embryogenese als Selbstkonstruktion}

Eine der faszinierendsten Fragen der Entwicklungsbiologie ist, wie sich ein Embryo von einem anfangs weitgehend ungeordneten Zellhaufen zu einem strukturierten Tier selbstorganisert. Wir wissen, dass eine Handvoll Signalmoleküle ausreicht, um das Zellorchester der Embryonalentwicklung zu dirigieren. Aber wie genau die räumliche und zeitliche Abfolge der Signale ohne äußere Einflüsse gesteuert wird, liegt immer noch weitestgehend im Dunkeln. Unsere Forschungsgruppe in Tübingen, zukünftig in Konstanz, verwendet einen interdisziplinären Ansatz mit quantitativen Messungen und mathematischer Modellierung, um diesen Fragen nachzugehen. Dabei liegt unser Fokus auf Signalmolekülen der TGF- $\beta$ Familie - Nodal und BMP - die für die Entwicklung aller Wirbeltiere essenziell sind [1-4]. Nodal und BMP können sogar einen nahezu vollständigen Embryo erzeugen, wenn künstliche Quellen dieser Signale in Stammzellen eingebracht werden [2, 5]. Dabei bilden sie Gradienten aus, die je nach Signalstärke unterschiedliche Gewebe hervorbringen und so ein Koordinatensystem für den zukünftigen Körperbauplan erzeugen (Abb. 1A).

Bereits vor fast 70 Jahren ersann Alan Turing ein Modell, wie sich Pärchen solcher 

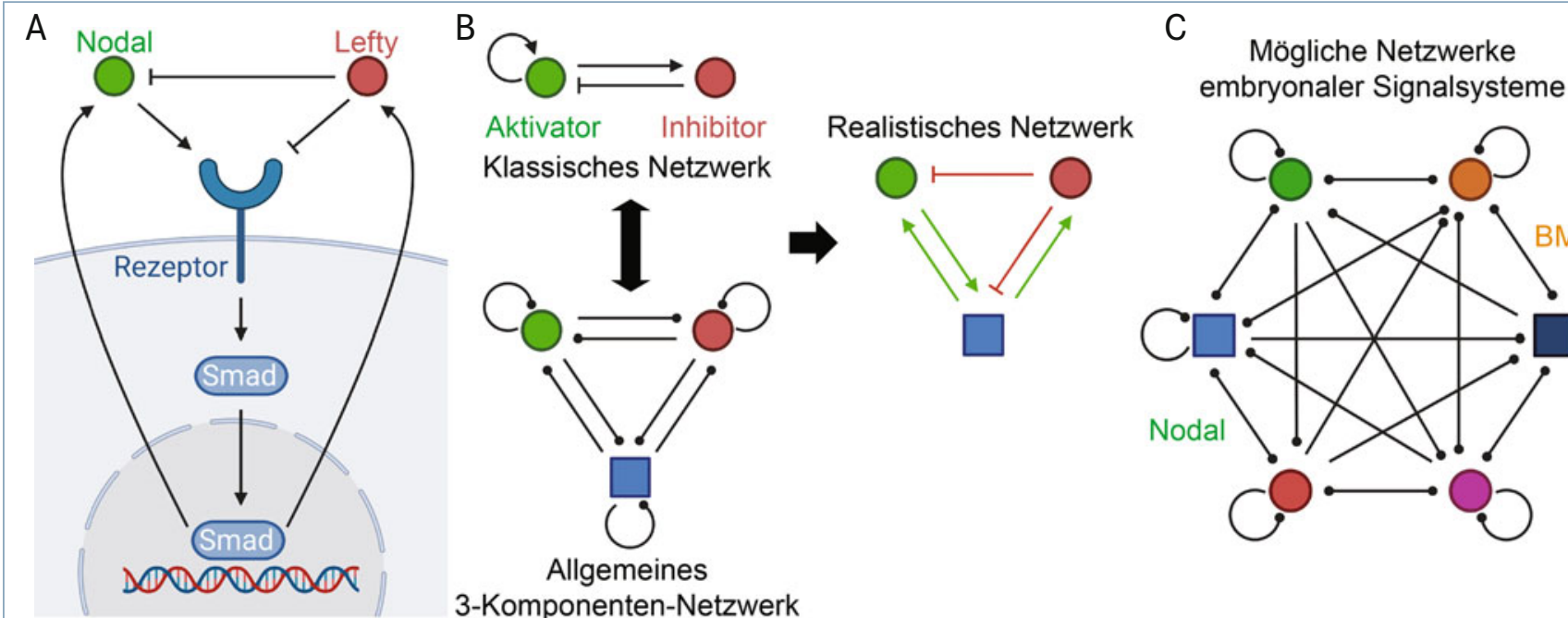

Klassisches Netzwerk
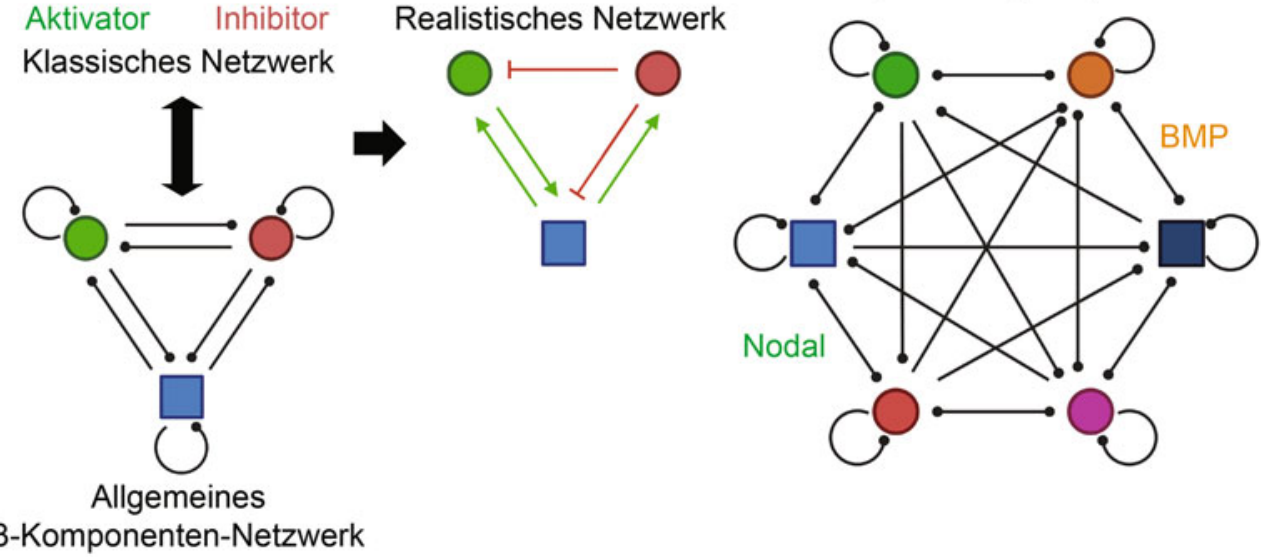

A Abb. 2: Selbstorganisation in Multikomponentennetzwerken. A, Nodal-Signaltransduktion: Das Signalmolekül Nodal bindet auf der Zelloberfläche an einen spezifischen Rezeptorkomplex, der daraufhin ein cytoplasmatisches Smad-Protein aktiviert. Das aktivierte Smad-Protein bindet dann im Zellkern spezifische regulatorische DNA-Elemente von Nodal-Zielgenen, wie beispielsweise Nodal und Lefty. Lefty selbst inhibiert die Nodal-Signaltransduktion, indem es an Nodal oder dessen Rezeptor bindet. B, Modellierung von selbstorganisierenden Multikomponentensystemen mit diffundierbaren (Kreise) und statischen (Quadrate) Molekülen. C, Modelle komplexer Multikomponentensysteme.

Signalmoleküle spontan in Gradienten und wellenförmige Muster selbstorganisieren könnten (Abb. 1B). Dass dies zu einer Zeit geschah, als Signalmoleküle noch nicht bekannt waren, ist beeindruckend. Turing nannte diese geheimnisvollen Pärchen Morphogene - Formgeber - [6], und später erkannten Hans Meinhardt und Alfred Gierer, dass sich Morphogene elegant als Aktivatoren und Inhibitoren verknüpfen lassen [7]. Turing, Meinhardt und Gierer betonten stets, dass in solchen einfachen Zweikomponentensystemen nur dann Muster entstehen könnten, wenn der Inhibitor eine viel größere Diffusionsrate als der Aktivator aufweist und der Aktivator nicht nur sich selbst verstärkt, sondern auch die Produktion des Inhibitors steuert. In diesem berühmten Modell der lokalen Selbstaktivierung und lateralen Inhibition verstärkt der Aktivator zunächst zufällig auftretende Fluktuationen, wodurch lokal sowohl mehr Aktivator als auch mehr Inhibitor gebildet werden. Der schnellere Inhibitor wandert dann zügig in angrenzende Regionen, um dort den Anstieg des Aktivators zu bremsen, sodass nur lokale Aktivatorspitzen entstehen können, die in kleinen Geweben zu Gradienten und in großen Geweben $\mathrm{zu}$ wellenförmigen Mustern führen (Abb. 1B).

\section{Morphogene, die Lenker der Selbstorganisation}

Durch embryologische Experimente und biophysikalische Messungen konnte gezeigt werden, dass der Nodal-Signalweg genau die Eigenschaften hat, die von Turing, Meinhardt und Gierer vorhergesagt worden waren: Nodal nimmt dabei die Rolle des sich selbstverstärkenden Aktivators ein, der die Produktion des viel diffundierbareren Inhibitors Lefty steuert (Abb. 1C, [8]). Die Wechselwirkung zwischen Nodal und Lefty führt dazu, dass der Signalweg nur in der Nähe der Nodal-Quelle - bei der Zebrafisch-Frühentwicklung ist dies die Marginalzone an der Grenze zwischen Dottersack und eigentlichem Embryo - aktiv ist. Dort steuert Nodal die Bildung von Mesendoderm, den Vorläuferzellen der inneren Organe, Blut und Muskeln (Abb. 1C). Wird der Dottersack samt Marginalzone früh in der Entwicklung entfernt, entsteht in diesen Explantaten durch polarisierte maternale Faktoren dennoch eine Nodal-Signalquelle, die zu einem Symmetriebruch sowie Mesendodermbildung führt [9]. Sogar vollständig dissoziierte und reaggregierte Zebrafischzellen können diesen Symmetriebruch hervorbringen (Abb. 1D, [10]) - ein erstaunliches Beispiel für die Fähigkeit des Embryos zur Selbstkonstruktion aus scheinbar chaotischen Anfangszuständen.

\section{Morphogene steuern auch die Größenregulation}

Das Nodal/Lefty-System aus kurzreichweitigem Aktivator und langreichweitigem Inhibitor kann noch mit weiteren erstaunlichen Leistungen aufwarten. Ein altbekanntes Phä- nomen der Entwicklungsbiologie ist die GröBenregulation: Selbst wenn man einen beträchtlichen Teil eines Embryos entfernt, kann sich das Gewebe zu einem Tier entwikkeln - und zwar gemäß seiner neuen Größe mit verringerten Proportionen aller Gewebe. Wir konnten kürzlich zeigen, dass die lange Reichweite des Inhibitors ein Vermessen der Embryogröße ermöglicht (Abb. 1E, [11]). Entfernt man einen großen Teil eines Zebrafischembryos, steigt die Konzentration des langreichweitigen Inhibitors Lefty an - ähnlich wie in einer verkleinerten Badewanne mit konstantem Wasserzufluss. Dabei wird der kurzreichweitige Nodal-Gradient von der Größenänderung nicht direkt beeinflusst. Die steigende Inhibitorkonzentration schmälert dann im Folgenden aber die Nodal-Aktivität, sodass weniger Mesendoderm, gemäß der neuen Embryogröße, gebildet wird (Abb. 1E).

\section{Selbstorganisation mit quantitativen Daten und neuen Modellen untersuchen}

Obwohl unsere Modelle die Phänomene während der Zebrafisch-Embryogenese gut beschreiben, sind die vereinfachten Zweikomponentennetzwerke nur eine Annäherung an die biologische Realität. Echte biologische Signalsysteme umfassen weitaus mehr Mitspieler als nur sezernierte Aktivatoren und Inhibitoren - insbesondere signalweiterleitende Moleküle wie membrangebundene Rezeptoren und Transkriptionsfaktoren, die aber die Zelle nicht verlassen und 
deshalb im Entwicklungskontext eines Gewebes als statisch gelten können (Abb. 2A). Turing, Meinhardt und Gierer konnten die Zweikomponentennetzwerke damals noch mit recht einfachen Mitteln wie Stift und Papier untersuchen, um die mathematischen Bedingungen für die Selbstorganisation zu definieren. Das funktioniert bei komplexeren Multikomponentensystemen, die auf jede erdenkliche Weise miteinander verschaltet sein könnten (Abb. 2B), nicht mehr. Starke Computer, die aber erst seit Kurzem zur Verfügung stehen, können da helfen. Bei unseren jüngsten Untersuchungen mit moderner Computerunterstützung zeigte sich überraschenderweise, dass realistischen Multikomponentennetzwerken fundamental andere selbstorganisierende Mechanismen zugrunde liegen als bisher angenommen worden war [12]. Die Bedingung, dass ein Inhibitor eine weitaus gröBere Diffusionsrate aufweisen muss als ein Aktivator, ist nicht so streng notwendig für die Musterbildung wie früher vermutet. Multikomponentensysteme mit beweglichen und statischen Molekülen können sogar mit gleicher Diffusionsgeschwindigkeit selbstorganisierte Muster ausbilden. Auch die Unterteilung in einfache Aktivatoren und Inhibitoren schlägt bei diesen komplexeren Systemen fehl. Vielmehr lassen sich die einzelnen Komponenten dieser neuen Netzwerke in Untermodule zusammenfassen (Abb. 2B): Untermodule mit insgesamt aktivierender Wirkung sind stabilisierend, jene mit hemmender Wirkung hingegen destabilisierend. Muster können sich dann spontan bilden, wenn die destabilisierenden Wechselwirkungen unter dem Einfluss der Diffusion die stabilisierenden Wechselwirkungen aus dem Gleichgewicht werfen. Wie bei Turing, Meinhardt und Gierer hängt die Musterbildung auch in komplexeren Systemen nach wie vor von dem Spannungsfeld zwischen Aktivierung und Inhibition ab, wenngleich nun der Effekt ganzer Untermodule zu berücksichtigen ist und die sezernierten Signalmoleküle die gleiche Diffusionsgeschwindigkeit aufweisen können - sogar langsame Inhibitoren und schnelle Aktivatoren sind in diesem neuen Rahmen möglich!

Wie funktionieren nun diese neuen Musterbildungssysteme genau? In klassischen Zweikomponentennetzwerken treibt die unterschiedliche Diffusionsrate das System von einem homogenen und stabilen Gleichgewicht weg, um Muster zu erzeugen. In Mehrkomponentennetzwerken wird dies durch die statischen, nicht diffundierbaren Netzwerkkomponenten erreicht, die kleine Störungen schnell verstärken können, da sie nicht dem homogenisierenden Effekt der Diffusion unterliegen und gleichsam als Kondensatoren wirken, die den Signaleintrag der diffusionsfähigen Moleküle integrieren.

\section{Embryologie 2.0 -}

Wechselwirkungen von

\section{Signalsystemen verstehen}

Ein großes Problem bei der Modellierung von Signalmustern ist die Komplexität biologischer Netzwerke, die in der Regel eine Vielzahl miteinander wechselwirkender Komponenten umfassen (Abb. 2C). Wie oben beschrieben werden die frühesten Musterbildungsentscheidungen von den sezernierten Signalmolekülen Nodal und BMP gesteuert. Diese wiederum werden von ihren eigenen Inhibitoren in Schach gehalten. Zusammen mit ihren intrazellulären Signaltransduktionskaskaden sind also alleine sechs Komponenten in einem möglichen Interaktionsnetzwerk zweier Signalwege zu berücksichtigen. Unter der Annahme, dass jedes Molekül sich selbst sowie auch alle anderen Moleküle beeinflussen kann, gilt es in diesem einfachen Fall bereits $6 \times 6=36$ mögliche Interaktionen zu berücksichtigen (Abb. 2C) - bei drei Signalwegen 81 Interaktionen, bei vier Signalwegen 144 usw. Dieser kombinatorischen Explosion ist auch mit der Unterstützung durch die oben erwähnten hochleistungsfähigen Computeranalysen nur schwer beizukommen, sodass wir neue Wege zur Analyse von Signalsystemen mit künstlicher Intelligenz beschreiten wollen.

Algorithmen der künstlichen Intelligenz haben in den letzten Jahren beeindruckende Fortschritte vollzogen, insbesondere im Erkennen und Generieren von Bildmustern. Wir wollen diese neuen Werkzeuge nutzen, um drei große Fragestellungen der Entwicklungsbiologie zu bearbeiten.

Erstens: Können wir künstliche Intelligenz nutzen, um Störungen bei der Musterbildung spezifischen Kombinationen von Signalmolekülen zuzuweisen? Die

\section{Hier steht eine Anzeige.}

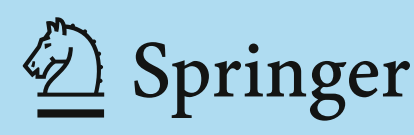




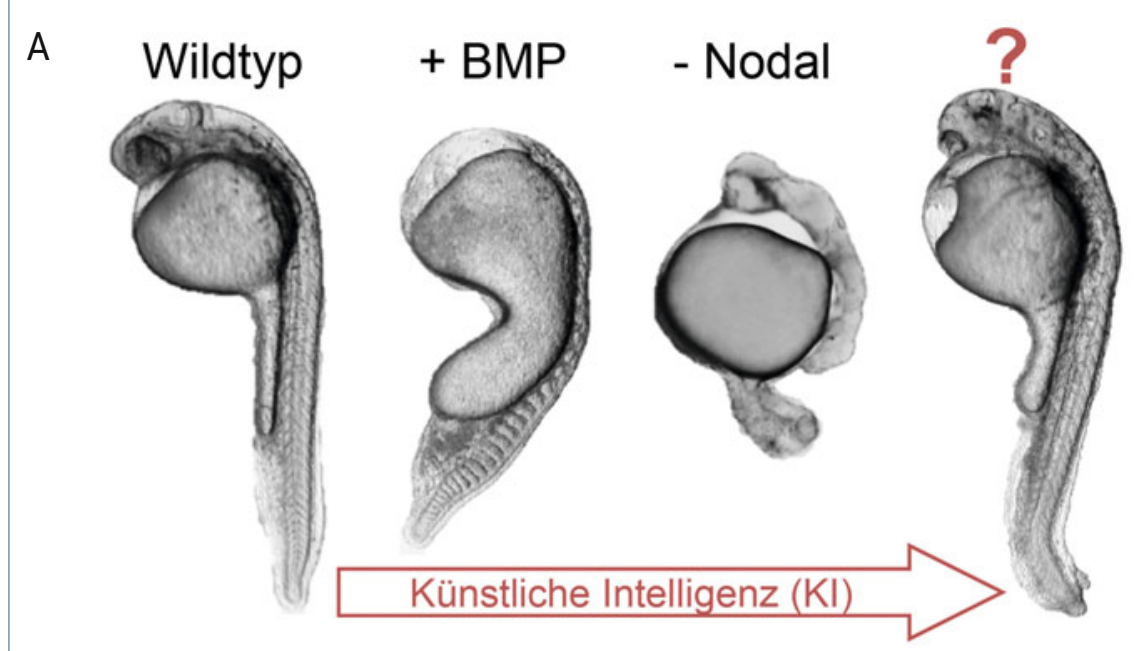

B

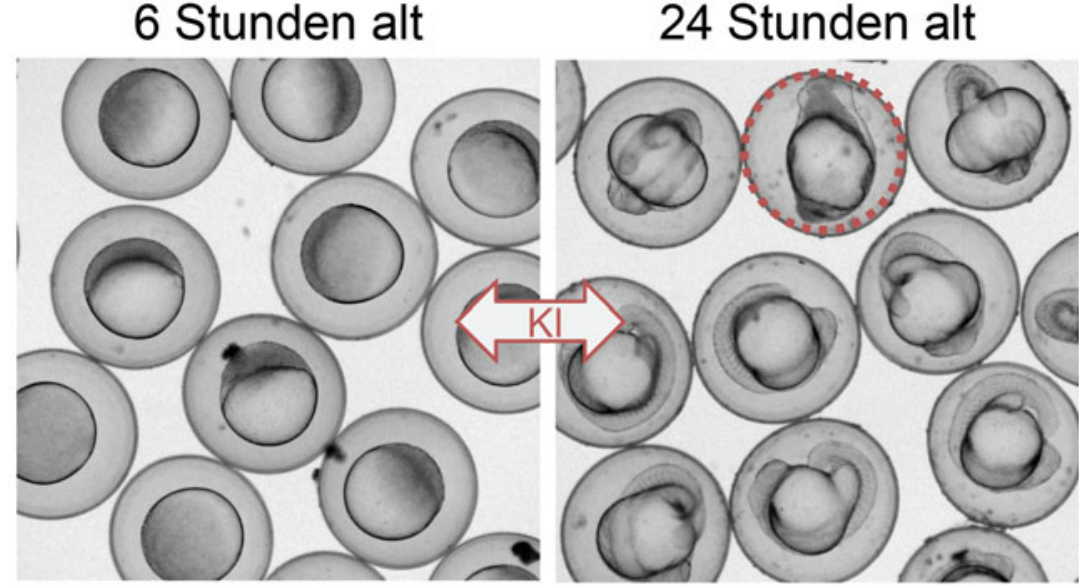

C

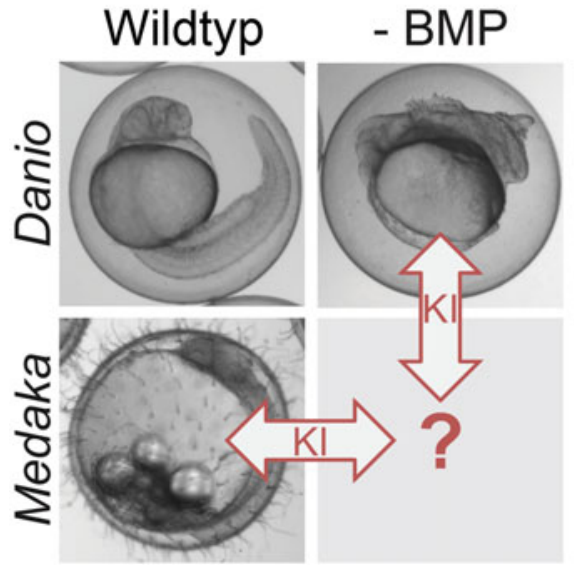

- Nodal

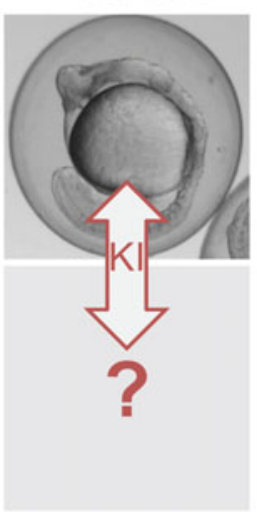

Abb. 3: Embryologie 2.0 - Große Fragen der Entwicklungsbiologie mit künstlicher Intelligenz untersuchen. A, Neue Phänotypen aus bekannten Phänotypen ableiten. B, Abweichler identifizieren. C, Vergleichende Embryologie - Morphologien morphen.

Betrachten der Bilder? Kann uns dieser Algorithmus möglicherweise sogar vorhersagen, wie Zebrafische aussehen würden, bei denen die Aktivität mehrerer Signalwege gleichzeitig ausgeschaltet wurde (Abb. 3A)?

Zweitens: Können wir künstliche Intelligenz nutzen, um Störungen bei der Musterbildung zu detektieren, bevor ein Mensch sie mit dem Auge sehen kann? Zebrafische entwickeln sich erstaunlich robust, aber bei einem geringen Prozentsatz kommt es zu Entwicklungsfehlern, die nach Fertigstellung des Körperbauplans gut zu erkennen sind. Diese Fehlbildungen müssen ihre Ursache in früheren Entwicklungsprozessen haben, können aber dort mit dem bloßen Auge nicht erkannt werden (Abb. 3B). Wenn wir maschinelles Sehen nutzen könnten, um diese Abweichler zu identifizieren, könnten wir die molekularen Ursachen für solche Fehlentwicklungen besser ergründen. Welcher Signalweg ist zuerst aus dem Ruder gelaufen? Ähnlich wie bei einer Lawine: Ein Stein ist der Auslöser, aber den Menschen im Tal ist es unmöglich, diesen mit dem bloßen Auge zu erkennen.

Drittens: Können wir künstliche Intelligenz nutzen, um zu verstehen, wie sich Embryonen verschiedener Tierarten entwickeln? Die Umgebung, in der Zellen sich entwickeln - der Embryo - ist in der Gestalt über Tierarten hinweg sehr variabel. Selbst Wirbeltiere haben eine erstaunliche Divergenz in ihrer Form. Wenn man einen Computer mit hinreichend vielen Daten verschiedener Spezies und korrespondierenden Signaldefekten speisen würde - könnten wir vorhersagen, wie Entwicklungen und Defekte in Embryonen mit einer anderen Geometrie aussähen (Abb. 3C)? Und lassen sich andersherum möglicherweise Rückschlüsse ziehen, ob Änderungen in der Aktivität bestimmter Signalwege für eine bestimmte Morphologie verantwortlich sind?

Dank der schnell voranschreitenden Entwicklungen im Bereich Bildgebung und künstlicher Intelligenz scheint die Zeit reif für „Embryologie 2.0“. Sie wird uns näher an ein mechanistisches Verständnis zur Natur der Selbstkonstruktion verschiedenster Embryomorphologien bringen.

Phänotypen einzelner defekter Signalwege sind beim Zebrafisch wohlbekannt und lassen sich durch Behandlung mit spezifischen niedermolekularen Substanzen gut rekapitulieren. Die Entwicklung des Körperbauplans ist bei Zebrafischen innerhalb von einem Tag abgeschlossen, und wir können tausende
Bilder in dieser Zeit mit Hochdurchsatzmikroskopen aufnehmen. Wenn es uns gelänge, einen Algorithmus auf die embryonalen Phänotypen der wichtigsten Signalwege zu trainieren - könnte dieser Algorithmus dann unbekannte niedermolekulare Substanzen einem Signalweg zuordnen, allein durch

\section{Danksagung}

Wir danken der Max-Planck-Gesellschaft, dem ERC (QUANTPATTERN - 637840 und ACE-OF-SPACE - 863952) und dem FWF (J 4507-B) für die Unterstützung unserer Arbeiten. 


\section{Literatur}

[1] Rogers KW, Müller P (2019) Nodal and BMP dispersal during early zebrafish development. Dev Biol 447: 14-23 [2] Soh GH, Pomreinke AP, Müller P (2020) Integration of Nodal and BMP signaling by mutual signaling effector antagonism. Cell Rep 31: 107487

3] Pomreinke AP, Soh GH, Rogers KW et al. (2017) Dynamics of BMP signaling and distribution during zebrafish dorsal-ventral patterning. eLife 6: e25861

[4] Rogers KW, ElGamacy M, Jordan BM et al. (2020) Optogenetic investigation of BMP target gene expression diversity. eLife 9: e58641

[5] Xu PF, Borges RM, Fillatre J et al. (2021) Construction of a mammalian embryo model from stem cells organized by a morphogen signalling centre. Nat Commun 12: 3277 6] Turing AM (1952) The chemical basis of morphogenesis. Philos Trans R Soc Lond B Biol Sci 237: 37-72

[7] Gierer A, Meinhardt H (1972) A theory of biological pattern formation. Kybernetik 12: 30-39

[8] Müller P, Rogers KW, Jordan BM et al. (2012) Differential diffusivity of Nodal and Lefty underlies a reaction-diffusion patterning system. Science 336: 721-724

[9] Schauer A, Pinheiro D, Hauschild R et al. (2020)

Zebrafish embryonic explants undergo genetically encoded self-assembly. eLife 9: e55190

[10] Fulton T, Trivedi V, Attardi A et al. (2020) Axis specification in zebrafish is robust to cell mixing and reveals a regulation of pattern formation by morphogenesis. Curr Biol 30: 2984-2994

11] Almuedo-Castillo M, Bläßle A, Mörsdorf D et al. (2018) Scale-invariant patterning by size-dependent inhibition of

Nodal signalling. Nat Cell Biol 20: 1032-1042

[12] Marcon L, Diego X, Sharpe J et al. (2016) High-

throughput mathematical analysis identifies Turing networks for patterning with equally diffusing signals, eLife 5: e14022
Funding note: Open Access funding enabled and organized by Projekt DEAL. open Access: Dieser Artikel wird unter der Crefiv 4.0 International Lizenz veroffentlicht, welche die Nutzung, Vervielfältigung Bearbeitung, Verbreitung und Wiedergabe in jeglichem Medium und Forma erlaubt, sofern Sie den/die ursprünglichen Autor(en) und die Quelle

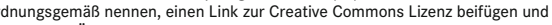
angeben, ob Änderungen vorgenommen wurden. Die in diesem Artikel . nichts anderes ergibt Sofern dis betreffende Material nicht unter der genannten Creative Commons Lizenz steht und die betreffende Handlung nich Wach gesetzlichen Vorschriften erlaubt ist, ist für die oben aufgeführten Weiterverwendungen des Materials die Einwilligung des jeweiligen Rechteinhabers einzuholen. Weitere Details zur Lizenz entnehmen Sie bitte der Lizenzinformation aur http://creativecommons.org/icenses/by/4.0/deed.de.
Korrespondenzadresse:

Prof. Dr. Patrick Müller

Friedrich-Miescher-Laboratorium der Max-PlanckGesellschaft

Max-Planck-Ring 9

D-72076 Tübingen

patrick.mueller@tuebingen.mpg.de,

patrick.mueller@uni-konstanz.de

www.fml.tuebingen.mpg.de/mueller-group

\section{AUTOREN \\ Daniel Čapek}

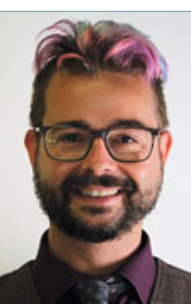

2000-2008 Keltologie-Diplom und 2007-2012 Zoologie- und Entwicklungsbiologiestudium an der Universität Wien, Österreich. 2018 Promotion am Institute of Science and Technology Austria (IST Austria). 2019-2021 Postdoc am Friedrich-MiescherLaboratorium in Tübingen. Seit 2021 als Erwin-Schrödinger-Stipendiat an der Universität Konstanz.

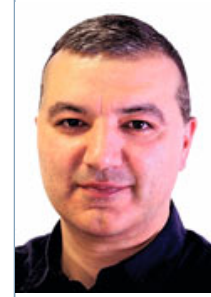

\section{Murat Ünalan}

1998-2004 Studium der Humanmedizin und Promotion an der Charité in Berlin 2004-2008 MD/PhD-Programm an der Medizinischen Hochschule Hannover (PhD), anschließend Postdoc/Arzt im Labor für Molekulare Hämatopoese und am Institut für Experimentelle Hämatologie. 2019-2021 Postdoc am Friedrich-Miescher-Laboratorium in Tübingen. Seit 2021 an der Universität Konstanz.

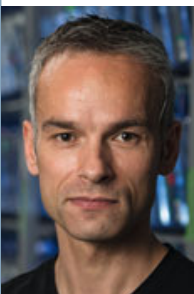

Patrick Müller

1999-2004 Molekularbiologie-Studium. 2007 Promotion am Max-Planck-Institut fü biophysikalische Chemie. 2007-2013 Postdoc an der Harvard University. 2013-

2021 Gruppenleiter und Professor am Max-Planck-Institut für Entwicklungsbiologie,

Friedrich-Miescher-Laboratorium \& Universität Tübingen. Seit 2021 Professor für

Entwicklungsbiologie an der Universitat Konstanz.
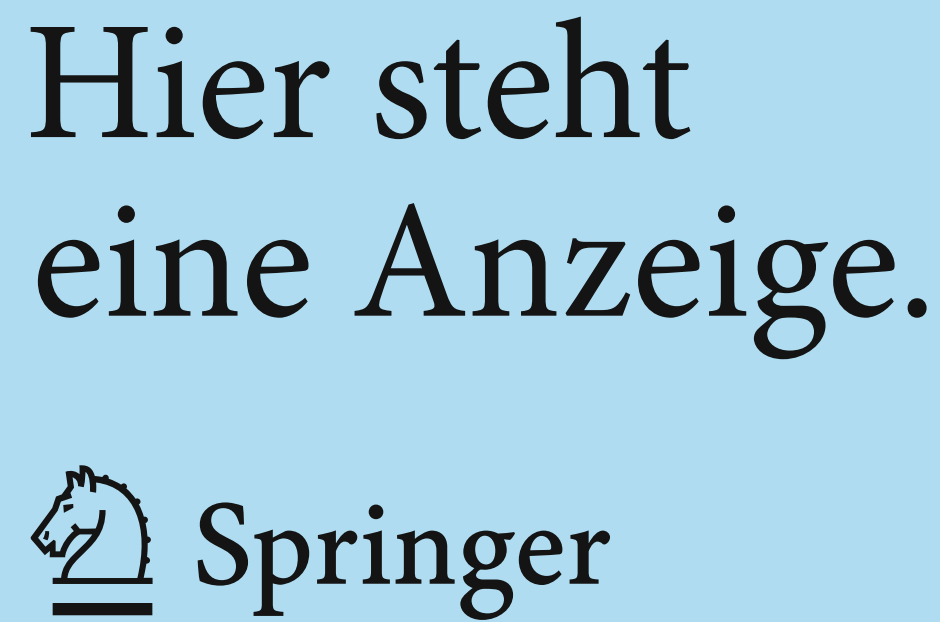